\title{
Die Katalogisierung der Bücher der ehemaligen Fideikommissbibliothek des Hauses Habsburg- Lothringen an der Österreichischen Nationalbibliothek
}

DOI 10.1515/bfp-2017-0030

Zusammenfassung: Die Bücher der Fideikommissbibliothek, die sich in ihrer historischen Aufstellung seit 1921 an der Österreichischen Nationalbibliothek befinden, wurden bis 2011 nur zu einem geringen Teil in den Bibliothekskatalog der Österreichischen Nationalbibliothek eingepflegt. Ziel der Österreichischen Nationalbibliothek ist es momentan, die Bestände der Bibliothek zur Recherche online verfügbar zu machen.

Schlüsselwörter: Österreichische Nationalbibliothek; Bibliotheksgeschichte

\section{Cataloguing the Books of the Former Fideicommiss Libra- ry of the House of Habsburg-Lothringen at the Austrian National Library}

\begin{abstract}
Although the Fideicommiss Library has been conserved as part of the Austrian National Library since 1921, the books have been incorporated only to a small extent in the Austrian National Library catalogues up to 2011. Currently, the Austrian National Library prepares access to the online cataloguing of the books of the Fideicommiss Library.
\end{abstract}

Keywords: Austrian National Library; history of libraries

\section{Die Vorgeschichte}

Die heutige Fideikommissbibliothek bildete bis zum Ende der österreichisch-ungarischen Monarchie im November 1918 einen Teil des fideikommissarisch verwalteten unteilbaren und unveräußerlichen Privateigentums der kaiserlichen Familie Habsburg-Lothringen. Fideikommissherren waren die jeweils Erstgeborenen in der männlichen Primogenitur der kaiserlichen Linie, die drei seit 1835 regierenden Kaiser von Österreich - mit einer Ausnahme, Erzherzog

*Kontaktperson: Mag. Michaela Ortner, michaela.ortner@onb.ac.at
Franz Karl 1875-1878. Das Kernstück der Familienbibliothek bildete die ehemalige Privatbibliothek und Sammlung des Kaisers Franz II. (I.) (1768-1835), ${ }^{1}$ und wurde in Folge jeweils durch die Privatbibliotheken der einzelnen Fideikommissherren erweitert. ${ }^{2}$

Im Jahre 1921 gelang die Habsburg-Lothringische Fideikommissbibliothek und ihre Spezialsammlungen ${ }^{3}$ nachdem sie bereits 1920 in die staatliche Verwaltung übernommen wurden - als „Porträtsammlung “4 an die Österreichische Nationalbibliothek. Gleichzeitig begann das Bundesministerium für Unterricht nach längerer Diskussion, gleichartige ehemalige kaiserliche Sammlungen - nun im Besitz der Republik - neu zu ordnen. ${ }^{5}$ Für die „Porträt-

1 Zur Gründungsgeschichte der Familien-Fideikommissbibliothek des Hauses Habsburg-Lothringen siehe Huber-Frischeis et al. (2015).

2 Ein Forschungsteam (Thomas Huber-Frischeis, Nina Knieling, Rainer Valenta) begann unter der Leitung von Dr. Hans Petschar, Direktor des Bildarchivs und Grafiksammlung mit der Aufarbeitung der Sammlungsgeschichte im Rahmen zweier vom Österreichischen Forschungsfonds (FWF) finanzierter Projekte. Das erste, abgeschlossene Projekt behandelte die Zeit der Gründung bis 1835. Das Folgeprojekt behandelt den Zeitraum 1835 bis 1921. Dazu wird im laufenden Jahr eine Publikation erscheinen. Mehr Informationen zu den Projektergebnissen finden Sie auf der Website des FWF-Forschungsprojekts unter http://fkb.onb.ac.at.

3 Im FKB-Archiv befindet sich ein Schreiben von Payer an das „Staatsamt für Inneres u. Unterricht“, wo er diese Spezialsammlungen (Porträtsammlung, Lavatersammlung, Handzeichnungen und graphische Blätter, Aquarelle, Büsten u. Reliefs sowie Huldigungsadressen) aufzählt und dafür plädiert, sie nicht zu trennen (25. Oktober 1920. FKBA47016). Das Archiv der Fideikommissbibliothek (1809-1945) hat sich im Bestand der Abteilung Bildarchiv und Grafiksammlung erhalten. Es verwahrt die Korrespondenz des Bibliothekars mit dem Kaiser und anderen nachgeordneten Hofstellen, bzw. mit zeitgenössischen Autoren und Künstlern. Derzeit ist der Bestand für die Jahre 1809-1921 erschlossen. Die Benutzung der Originalakten gleicht jenen des Archivs der ÖNB und ist im Lesesaal der Abteilung Bildarchiv und Grafiksammlung möglich.

4 Der Name der Sammlung wechselt des Öfteren bis heute. 1921 Porträtsammlung, ab 1946 Bildarchiv und Porträtsammlung, seit 2010 Bildarchiv und Grafiksammlung.

5 Stummvoll (1968) $580 \mathrm{ff}$. sowie Beilage IX, 622ff. 


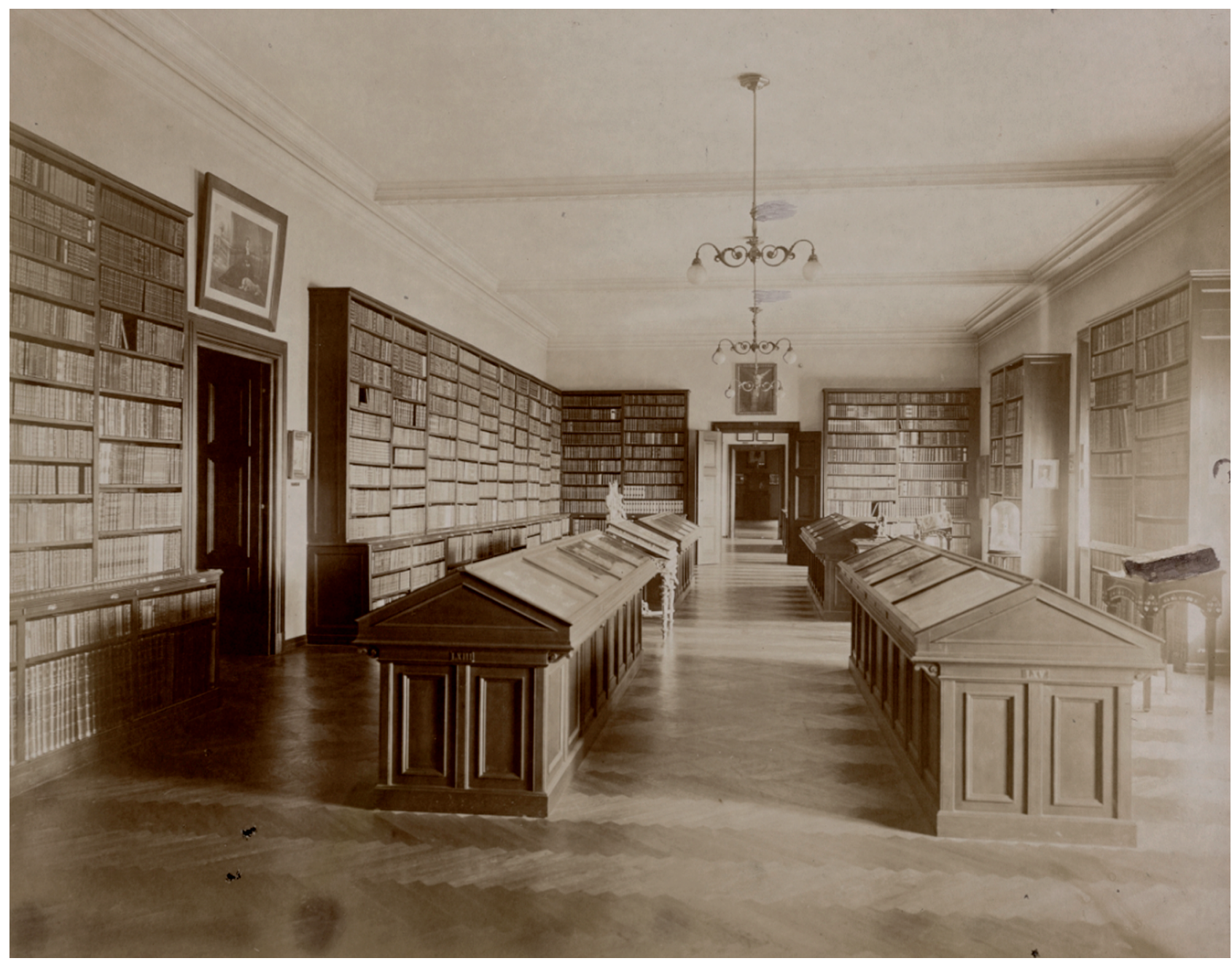

Abb. 1: Fideikommissbibliothek, Franz Josef Saal Aufnahme von Hermann Heydenhauss, um 1920

sammlung“ der Österreichischen Nationalbibliothek stellte diese Neuordnung einen Gewinn dar, erhielt sie aus dem Kupferstichkabinett der ehemaligen Hofbibliothek, aus der Intendanz der Hoftheater, aus dem Kriegsarchiv oder auch aus der Albertina eine große Anzahl an Porträts - u. a. auch die Bildnissammlung Prinz Eugens - überschrieben. Im Gegenzug wurden aus der ehemaligen Fideikommissammlung Kunstblätter, die keine Personen darstellen, Städteansichten, Handzeichnungen und Aquarelle an andere Sammlungen abgegeben. ${ }^{6}$

Auch die Handschriften, die Kartensammlung, geographische Werke, Inkunabeln, Musikalien und Papyri wurden auf diverse Abteilungen der Nationalbibliothek verteilt. ${ }^{7}$ Der seit 1908 genutzte Standort der Fideikommissbibliothek im zweiten Stock des Corps de Logis der Neuen Burg blieb erhalten.

\section{Die heutige} Fideikommissbibliothek ein „Corpus mortuum“?

Durch die finanziell schwierige Lage des Staates wurde die Nationalbibliothek nur mit einer sehr geringen Bücherdotation für die Erwerbung von Werken ausgestattet. Beschlossen wurde, durch Dublettenabgleiche von Werken der Fideikommissbibliothek und der Nationalbibliothek Werke zum Tausch und Verkauf zu gewinnen. Man erhoffte sich, durch den Erlös aus Dublettenverkäufen eine Weiterführung des Büchereinkaufes sichern zu können. So übernahmen Antiquare Dubletten und boten sie im Handel an. Unter anderem wurde hierfür auch ein Konto in Schweizer Franken bei Gilhofer und Ranschburg in den 
Jahren 1923 bis 1926 eingerichtet. ${ }^{9}$ Ein Verkaufskatalog wurde angelegt. Für das Frontispiz des Kataloges wählte man eigens ein mehrbändiges Prachtwerk aus der ehemaligen Privatbibliothek des Kaisers Franz II. (I.),$^{10}$ welches im gedruckten Katalog der Fideikommissbibliothek ${ }^{11}$ (Bd. I Sp. 323) mit der Bücherzahl 10119 verzeichnet und im Standortverzeichnis mit einem Stempelvermerk versehen war: abgegeben an die NB 2/23. Zudem wurde im Vorwort des Katalogs ausdrücklich darauf hingewiesen, dass es sich um die Privatsammlung des ehemaligen Kaisers handelt. $^{12}$

Der letzte Bibliothekar der Fideikommissbibliothek und infolge der erste Direktor der neuen „Porträtsammlung“ Payer von Thurn (1867-1932) lässt in seinem Aufsatz „Ein kaiserlicher Bibliophile“ 1927 die Leidenschaft des Sammlers Franz I. Revue passieren und endet mit der Geschichte der Zerstörung „dieser Lieblingsschöpfung des Kaisers Franz“ “. ${ }^{13}$ Er berichtet von den oben erwähnten Abgaben der Bestände an andere Abteilungen der Nationalbibliothek, aber auch, dass die Vorstände anderer staatlicher Sammlungen durch ein Rundschreiben eingeladen wurden, sich Werke aus der Bibliothek zu nehmen. Letztendlich hält er erstaunt fest, dass „verhältnismäßig wenig davon Gebrauch gemacht" ${ }^{14}$ wurde. Die meisten Lücken wurden in die Bestände aus der frühen Zeit der Bibliothek gerissen. Heute lässt sich darüber nur mutmaßen, ob dies damit zusammenhing, dass die gesamten Bestände schlecht bis gar nicht für Außenstehende recherchierbar, jene bis 1877 aber zumindest im Beckerkatalog erfasst waren.

\section{Die Recherchemöglichkeiten zu den Druckschriften der Fideikommissbibliothek}

Im Herbst 2010 versuchten wir, uns in der nunmehr zuständigen Sammlung der Österreichischen Nationalbibliothek, Bildarchiv und Grafiksammlung, einen Überblick über den Druckschriftenbestand der Fideikommissbiblio-

\footnotetext{
9 Vgl. ÖNB-Archiv, NB 1098/1923. Aber noch in den folgenden Jahren wurden Listen der abgegebenen und im Tausch erhaltenen Bücher geführt. NB 250 u. 966/1933; NB 20, 40, 55 u. 88/1934; NB 15 u. 120/ 1935; NB 91, 133, 195 u. 561/1936; NB 48, 62 u. 168/1937. Siehe Stummvoll (1973) 32.

10 Gilhofer und Ranschburg (1923).

11 Becker (1873).

12 Gilhofer und Ranschburg (1923) Vorwort.

13 Payer von Thurn (1927) 96.

14 Payer von Thurn (1927) $96 \mathrm{f}$
}

thek zu machen, was gar nicht so einfach war: So existiert eine Beschreibung der Werke der Fideikommissbibliothek in ihrer quantitativen Verteilung nach Sprachen und Disziplinen aus 1992 von Walter Wieser, dem damaligen Direktor der Porträtsammlung und des Bildarchivs. Allerdings hält er fest, dass sich die Titelzahlen ausschließlich auf die Druckschriften der Fideikommissbibliothek, die der Becker'sche Real- und Nominalkatalog verzeichnet hat, beziehen. ${ }^{15}$

Der erste Katalogband des Becker'schen Real- und Nominalkatalogs erscheint 1873 und beginnt mit den Abteilungen „Manuscripta“, „Incunabula“. Erst dann folgen die in alphabetischer Reihe angeordneten 30 Fächer der „Bücher nach dem Jahr 1530“. Innerhalb der Systematik werden die Werke chronologisch verzeichnet. Zur Unterscheidung der Werke aus der Fideikommiss- und der damals noch nicht zum Fideikommiss gehörenden kaiserlichen Privatbibliothek erhalten die Bestände Kaiser Franz Josef einen Stern vor der Bibliothekszahl. Durch die Erbschaft der umfassenden ferdinandeischen Privatsammlung ${ }^{16}$ aus Prag 1875 ergibt sich zudem die Notwendigkeit, den Aufbau des Katalogs zu ändern und einen weiteren, zusätzlichen Band $\mathrm{zu}$ planen. Die ferdinandeischen Bestände - nunmehr praktisch ein Teil der Privatbibliothek Franz Josefs - erfordern einen zusätzlichen Band. II/ 2. Mit diesem im Jahr 1879 erscheinenden Band II/2. sind nunmehr alle Bücher, die bis Juni 1877 in die Bibliotheken gelangten, verzeichnet. ${ }^{17}$ Die einzelnen Bände des Katalogs werden jeweils in einer geringen Auflage gedruckt und an sämtliche Familienmitglieder, diverse wichtige Persönlichkeiten und Institutionen aus Politik, Wissenschaft und Kultur verschenkt. Alle Werke die nach 1877 an die nun vereinte Familienbibliothek ${ }^{18}$ gelangten, wurden nach der Systematik des Beckerkatalogs in einem hand-

15 Wieser (1994) 152.

16 Erbschaft der Privatbibliothek Kaiser Ferdinands 1875. Durch den Umzug Ferdinands nach dessen Abdankung war diese imposante Privatsammlung 1850 zwischenzeitig in die Prager Burg zum Leidwesen des damaligen Fideikommissbibliothekars Wilhelm Leopold Khloyber mitübersiedelt worden. Vgl. dazu Akten aus dem FKB-Archiv: FKBA26004, FKBA26014.

17 Becker (1873), Becker (1875), Becker (1879) sowie Becker (1882).

181878 gelangt nunmehr Kaiser Franz Josef offiziell auch in den Besitz der familieneigenen Fideikommissbibliothek. Per Handschreiben vom 27.3.1878 verfügt der Kaiser die Vereinigung seiner Privatbibliothek und der Fideikommissbibliothek (FKB-Archiv, FKBA28082). Die separate Nummerierung in den Inventaren der bis dato verzeichneten Werke aus seiner Privatbibliothek blieb aufrecht. Die Privatbibliothek Kaiser Franz Josef mit ihren 8604 Werken wird somit geschlossen und wird als die „ferdinandeische Reihe“ bezeichnet. Ab diesem Zeitpunkt werden alle neu eingelangten Werke mit der fortlaufenden Bücherzahl der sog. „franziszeischen“ Reihe der „k. u. k. Familien- 
schriftlichen Zettelkatalog verzeichnet. Ebenfalls existiert ein handgeschriebener alphabetischer Katalog in 24 Foliobänden, der sog. Catalogus alphabeticus. Dieser musste durch den Zuwachs infolge des ständigen Anwachsens des Bestands der Bibliothek - durch leere Seiten interpoliert mehrmals umgebunden werden. Bei Recherchen der Sammlung zum Bestand der Druckschriften konnte er nur von Eingeweihten benutzt werden. Dieser Nominalkatalog mit systematischen und Schlagworthinweisen verzeichnet zudem nur die Titel der franziszeischen Reihe der Fideikommissbibliothek. Zusammen mit der Einlaufkonkordanz (10 handschriftliche Bände) bildet er die große Familienbibliothek ab, in der 48750 Titel verzeichnet sind.

Bereits im November 1964 startete man mit dem ersten Versuch die Fideikommissbibliothek, in den „Alten Katalog“ das Verzeichnis der Österreichischen Nationalbibliothek ihrer Werke mit den Erscheinungsjahren von 1501 bis $1929^{19}$ einzuarbeiten. Für den Druckschriftenbestand der Fideikommissbibliothek wurden dafür die Numerus-Currens-Signaturen 250.001 bis 290.000 reserviert. $^{20}$

In einem ersten Anlauf wurde dabei alphabetisch nach den Beschreibevorschriften der Hofbibliothek von 1901 vorgegangen..$^{21}$ Der erste Eintrag ins Einlaufbuch für die Fideikommissbibliothek am 16.11.1964 beginnt mit „A“ mit der Signatur 250001-D und lautet (Abelin, Johannes Philippus): De Gedenkwarrdige en al-om beroemde Voyagien der Spanjaarden, 1492 und endet mit „B“ La FerteSenectere - Appendice aux reflexions de M. Bergasse mit der Signatur 251128-B. Das Ordnungswort leitete sich in diesem Falle vom Namen Bergasse, [Nicolas] ab. Als Vor-

Fideikommiß-Bibliothek“ versehen, die zum damaligen Zeitpunkt 33924 Titel zählte.

19 Dieser „Alte Zettel-Katalog“ war nur in einem Exemplar im Haus vorhanden und daher für die Benutzung für das Publikum nicht geeignet. Katalogisiert wurde weiterhin nach der österreichischen Vorschrift von 1901. Die Abschreibungen des Alten Nominal-Kataloges wurden auf Initiative von Stummvoll ab 1958 im Hause mit fünffachen Mehrfachschreibanlagen begonnen. Die Abschrift der großformatigen Zettel auf ein kleineres internationales Kartenformat verlangte eine Kürzung der einzelnen Teile der Titelaufnahme. Aufzählungen der einzelnen Band- und Stücktitel bei Gesamtausgaben und Reihen wurden aus raumsparenden Gründen weggelassen. Vgl. Stummvoll (1973) 203.

20 Der Numerus Currens wurde bereits 1899 in der Hofbibliothek zuerst für Zeitschriften dann auch für Werke mit Erscheinungsjahr 1501-1929 eingeführt. Ausgenommen der Werke mit Standort Prunksaal. Dafür wurden auch sog. Einlaufbücher angefertigt und händisch ausgefüllt. Je ein Einlaufbuch enthält 10.000 Signaturen und im Jahre 1899 begann die fortlaufende Signatur mit 400.000. Die Signaturen 1 bis 399.999 waren für Umsignierungen der älteren Werke vorgesehen. Vgl. Mittendorfer (1994) 122.

21 Vgl. Geyer (1901). bereitung für diese Katalogisierung wurde anhand des handschriftlichen Alphabetischen Kataloges der Porträtsammlung sowie des gedruckten Beckerkataloges eine (im Wesentlichen) durchlaufende alphabethische Liste erstellt. Dabei wurden aber nur Werke herangezogen, die nicht bereits im Alten Katalog der Nationalbibliothek verzeichnet waren. Gleichzeitig zu dieser Katalogisierung vermerkte man die neu vergebenen Numerus-Currens-Signaturen der Druckschriftensammlung im Alphabetischen Katalog der Porträtsammlung.

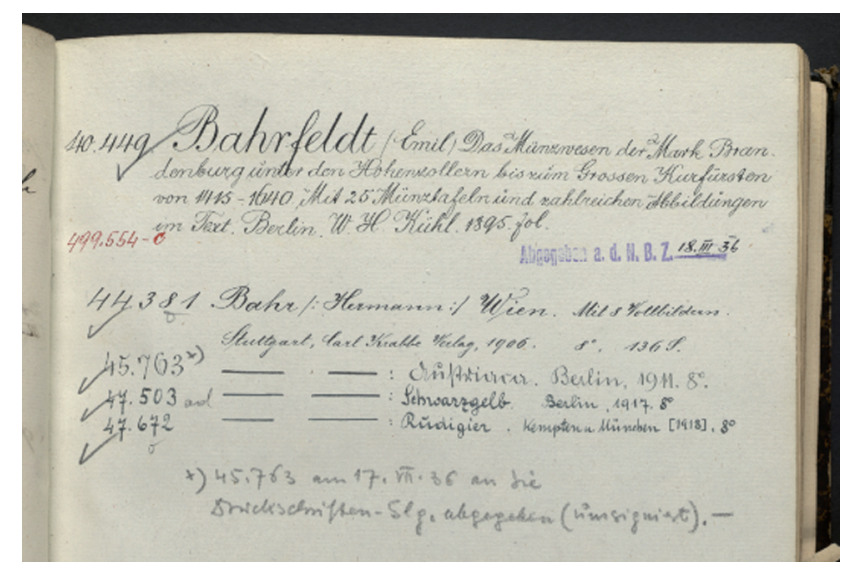

Abb. 2: Handschr. Alphabetischer Katalog der Porträtsammlung Bd. 2. BAA - Bibl

Dies stellte sich als ein sehr mühsames und langwieriges Unterfangen heraus, weil gleiche Werke in ihrer Beschreibung im Beckerkatalog sowie im handschriftlichen Katalog der Porträtsammlung im Vergleich zum Alten Katalog der Nationalbibliothek unter völlig verschiedenen Ordnungsworten erscheinen..$^{22}$ Betraut war mit diesem Katalogisierungsprojekt ein Frl. Stejskal. Letztendlich brach man die Vorgangsweise, die Werke in alphabetischen Listen zu erfassen und ausheben zu lassen, bereits $1966 \mathrm{ab}$.

In Folge wurden daraufhin systematisch nach der Kastenaufstellung beginnend bei Kasten 1 jene Titel ausgewählt, die im Haupthaus (Alter Katalog) nicht vorhanden waren. So wurden nur etwa $50 \%$ Werke in etwa 11000 Titel der Fideikommissbibliothek in den Kästen von 1-110 erfasst und sodann wurde das Vorhaben abgebrochen. ${ }^{23}$ Weitere 730 Titel, die Bestände des 16. Jahrhunderts, wur-

22 Im Bildarchiv und der Grafiksammlung wurden die Dienstzettel/ Korrespondenzen in Ordner gesammelt. So auch ein schriftlicher Schlagabtausch zwischen Trenkler und Pauer (?) vom 17.11.1964 und 18.XI.1964, wo es um die Umsignierung, Entlehnung und Haftung was den Transport der Werke der FKB in die Räume der Bearbeitung und Adjustierung im Haupthaus angeht.

23 Stummvoll (1973) 204. 


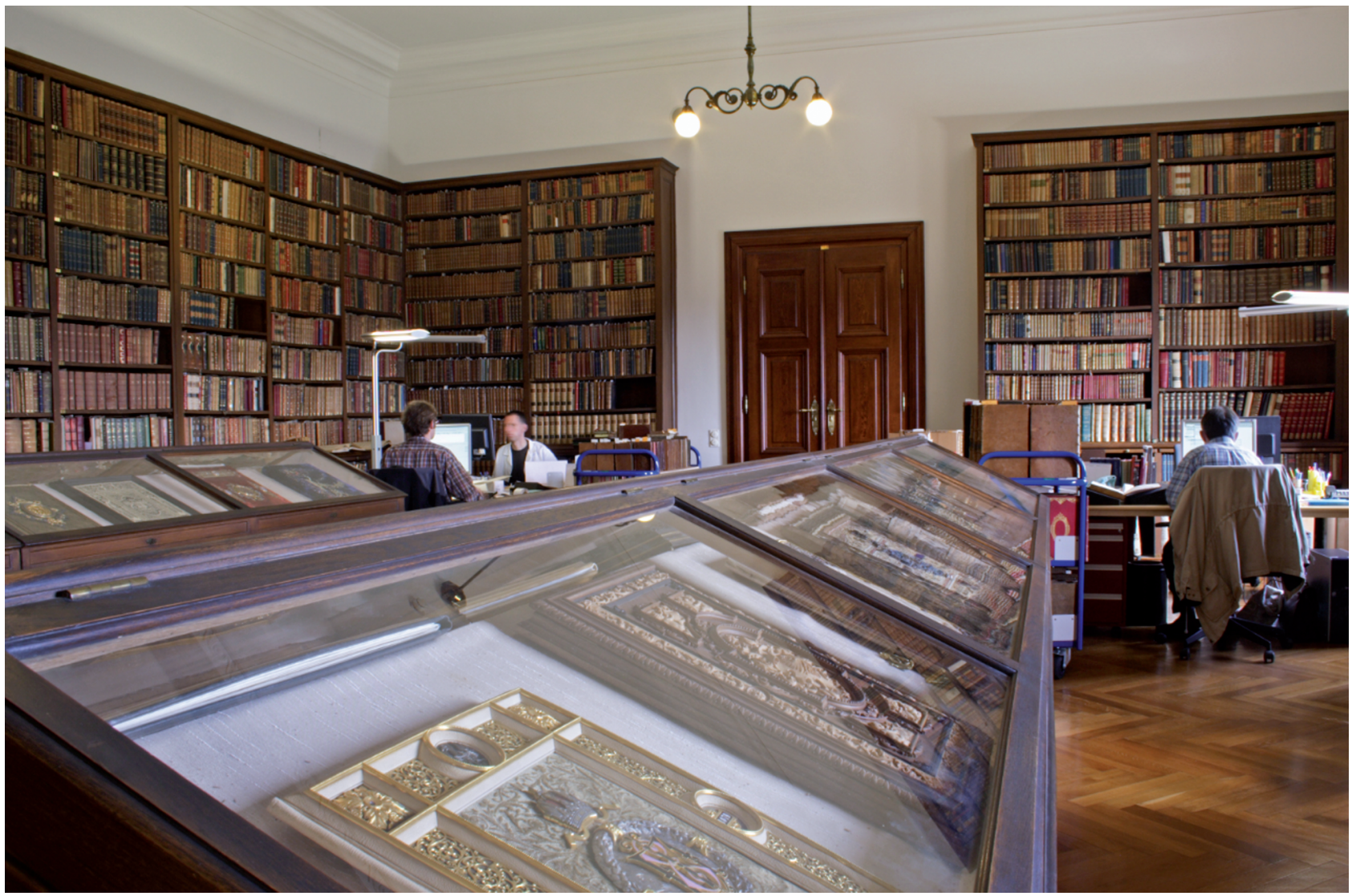

Abb. 3: Fideikommissbibliothek, Franz Josef Saal, Katalogisierung vor Ort 2012

den im Laufe der folgenden Jahre für das VD 16-Projekt gezogen und bearbeitet.

Ab Dezember 1997 wurde ein erneuter Katalogisierungsanlauf in der Porträtsammlung gestartet. Die Bestände sollten nunmehr in BIBOS aufgenommen werden. So setzte sich der erfasste Bestand der Fideikommissbibliothek innerhalb der ONB-Kataloge im Jahre 2010 wie folgt zusammen:

- ehem. ONB02 (1501-1929): 16393

- ehem. ONB03 (1930-1991): 2

- ehem. ONB01 (1992ff.): 2410

Im Hauptkatalog der Österreichischen Nationalbibliothek fanden sich aus der Familienbibliothek zum damaligen Zeitpunkt lediglich 18805 Werke verzeichnet. Obwohl seit 1921 zur Bibliothek gehörig, schien der Großteil des Bestandes aufgrund ihrer mangelhaften Erschließung in überregionalen und über Internet weltweit recherchierbaren Verzeichnissen nicht auf.

\section{Das Katalogisierungsprojekt}

Das ursprüngliche Ziel des derzeit noch laufenden internen Katalogisierungsprojektes ${ }^{24}$ sollte die Sichtbarmachung des noch verbliebenen wertvollen Bestandes der Fideikommissbibliothek im eigenen „Alten Katalog“ ONB02 (15011929) der Österreichischen Nationalbibliothek sein. Gleichzeitig sollten künftig die Werke bis zum Erscheinungsjahr 1878 als Digitalisate den Lesern zur Verfügung gestellt werden. ${ }^{25}$ Ein weiteres Ziel wäre infolge auch eine virtuelle Rekonstruktion des Gesamtbestandes der ehemaligen Fideikommissbibliothek ${ }^{26}$ samt der Möglichkeit, die in der Familienbibliothek vereinten Privatbibliotheken der einzelnen Fideikommissherren einzeln darzustellen. Dafür begannen wir 2011 ein Projektteam zusammenstellen, dass

24 Bis Ende Juni 2017.

25 Im Rahmen des ABO-Projekts sollten die ersten Werke digitalisiert werden. In einer Public Private Partnership mit Google digitalisiert die Österreichische Nationalbibliothek ihren Buchbestand vom frühen 16. Bis zum späten 19. Jahrhundert.

26 Eine virtuelle Bibliothek inklusive der abgegebenen Bestände in die Kartensammlung, Musiksammlung, Papyrussammlung und Sammlung von Handschriften und Alten Drucken. 
aus zwei Bibliothekarinnen aus der Sammlung Bildarchiv und Grafiksammlung und zwei Projektmitarbeitern für die Katalogisierung, sowie eine Projektmitarbeiterin für die Buchsicherung besteht. Durch die Erfahrungen aus den früheren Katalogisierungsprojekten beschlossen wir, die Werke nach der Kastenaufstellung zu bearbeiten.

Die Bücher werden ausgehoben, gereinigt und per $\mathrm{Au}$ topsie nach Standard der RAK-WB Alte Drucke in der ONB02 erfasst. Triff man auf vorhandene Katalogisate nach der ehemaligen Beschreibevorschrift von 1901, werden diese durch Eingabe von fehlenden Titelangaben ergänzt und so erhalten, z. B. mehrbändige begrenzte Werke und Reihen Stücktitel. Personennamen wurden am Anfang noch mit der damals gültigen PND verknüpft. Vermerkt wird im Katalogisat auch die historische Bücherzahl, die sog. Beckernummer, um das Werk der ferdinandeischen oder franziszeischen Reihe zuordnen zu können. Ehemalige handschriftliche Besitzvermerke auf Titelblatt oder Vorsatz sowie handschriftliche Widmungseinträge aber auch eingeklebte Exlibris werden erfasst. Letztendlich erhält jedes Werk einen Barcode und neben der Numerus-Currens-Signatur eine zweite Signatur bestehend aus der Kastennummer und der Nummer der Aufstellung innerhalb des Kastens. Die leeren Seiten der Einlaufbücher werden mit Kurztitel befüllt (Stand Oktober 2016 bei 286.596-C). Letztendlich wurden wir im Rahmen des Projektes öfter mit Änderungen der Katalogisierungsvorgaben konfrontiert. Es begann mit der Einführung des neuen Bibliotheksportal QuickSearch, der Umstellung auf die GND und der größten Veränderung durch die Migration der lokalen ONB02 in die ACC01. Ab Februar 2012 musste nun die Katalogisierung im Verbund (ACC01) durchgeführt werden. Dies erfordert einen komplexeren Ablauf bei der Katalogisierung, weil nun in zwei Datenbanken gearbeitet werden muss.

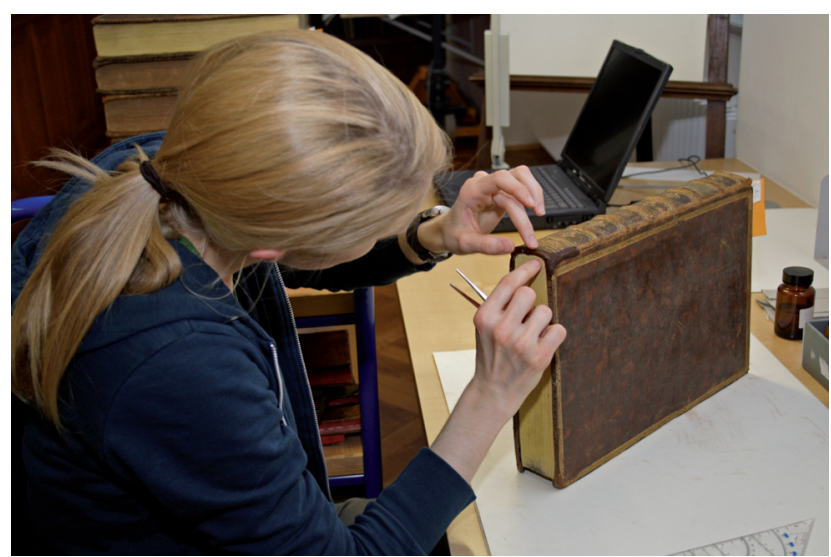

Abb. 4: Bearbeitung eines Einbandes mit Japanpapier
Einige Bücher in der Bibliothek haben im Laufe der Zeit Schäden erlitten. Leichte und mittlere Einbandschäden werden mit eingefärbtem Japanpapier gesichert. Risse in den Seiten und lose Blätter werden direkt in der Sammlung erstversorgt. Für dünne Broschüren und Stoffeinbände werden Umschläge angefertigt. Schwere Fälle werden markiert und vom Institut für Restaurierung der Österreichischen Nationalbibliothek falls möglich behandelt. Ungelesene Werke mit nicht aufgeschnitten Seiten erhalten im Katalogisat einen Vermerk und werden je nach Möglichkeit aufgeschnitten.

\section{Unerwartete Funde}

Erstaunlicherweise finden sich in einigen Werken noch Widmungsbriefe, Gesuche an den Kaiser von Autoren oder Verleger, die nicht von den Bibliothekaren im Fideikommissarchiv abgelegt wurden. Teilweise wurden zudem schlichte Briefe, mitunter sehr aufwendig gestaltet, gefunden.

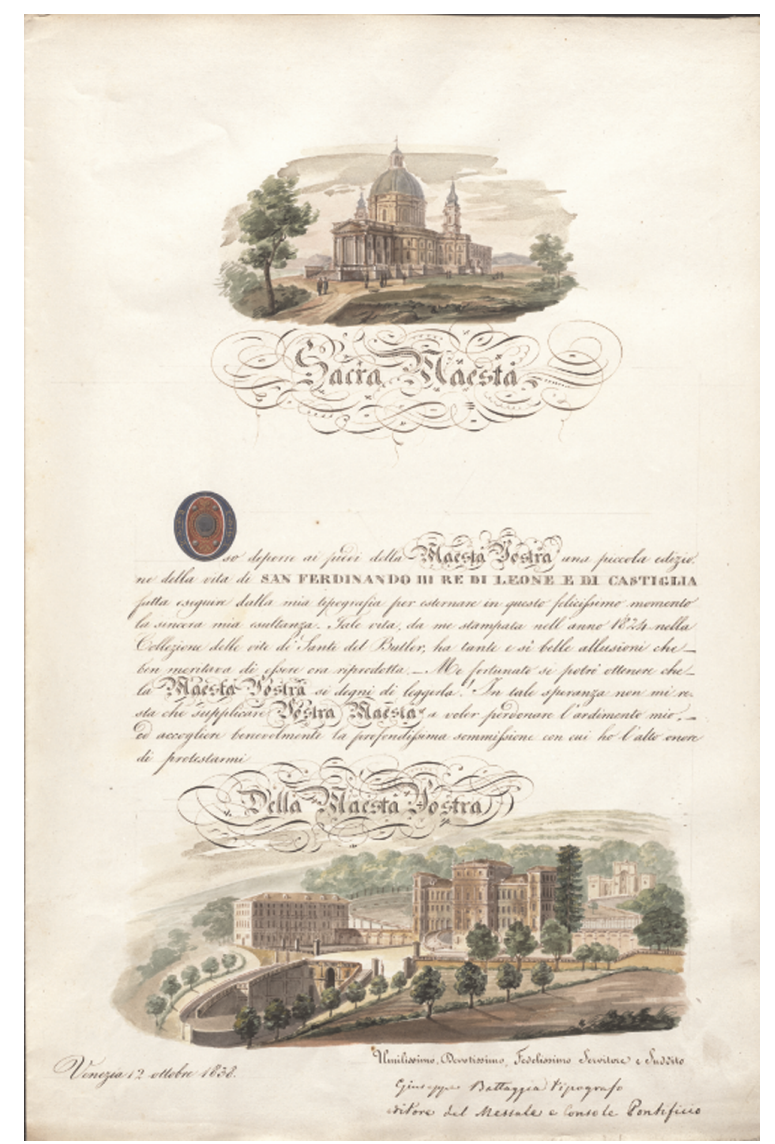

Abb. 5: Widmungsbrief des Typografen Guiseppe Battaggia an Seine Majestät datiert mit Okt. 1838 aus der ferdinandeischen Reihe *4458 mit Aquarellen versehen. Signatur: 286.492-D 
Eingelegte Briefe, Notizen, Lesezeichen etc. werden aus den Büchern entnommen und jeweils mit der Signatur des Werkes versehen. Fragile Objekte, wie z. B. ein selbst angefertigtes Lesezeichen, erhalten eine eigene säurefreie Hülle und der Fundort im Buch wird mit der Angabe der Seitenzahl vermerkt.

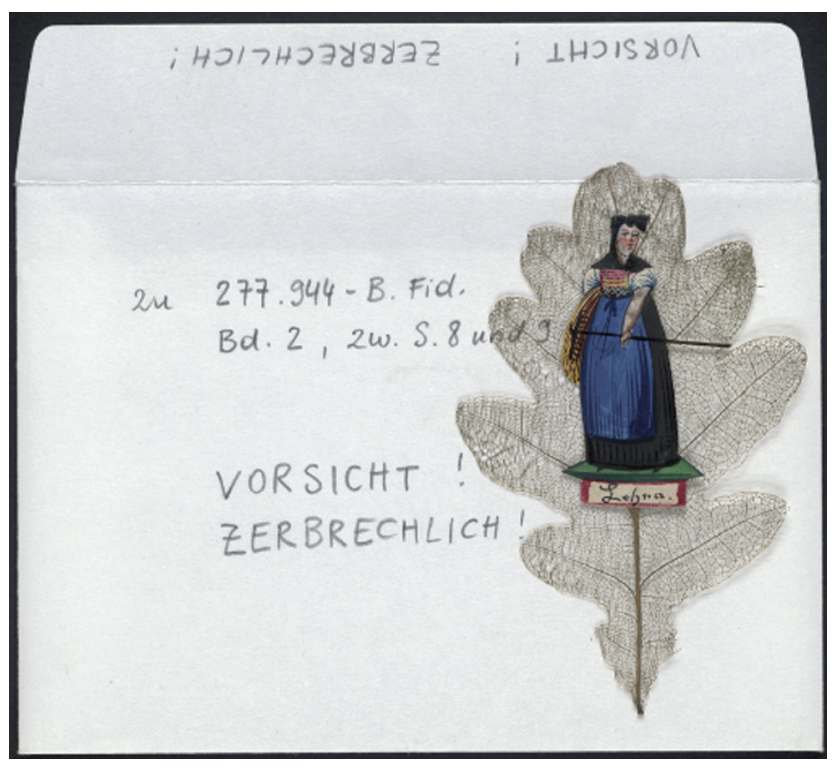

Abb. 6: Filigranes Lesezeichen aus „Briefe aus allen Jahrhunderten der christlichen Zeitrechnung. Zweyte Sammlung“ von Sailer, Johann Michael. Teil der ferdinandeischen Reihe *6234. Bd. 2, S. 8/9 Signatur: 277.944-B.2

Solcherart von Funden neben handschriftlichen Anmerkungen, Unterstreichungen Besitzvermerke, aber auch nicht aufgeschnitten Seiten von Werken können einiges über ihre einstigen Besitzer und Leser dieser Bibliothek erzählen. Hier ist noch vieles aufzuarbeiten.

\section{Literatur}

Becker, Moritz Alois Ritter von (1873): Die Sammlungen der vereinten Familien- und Privatbibliothek Sr. M. des Kaisers. Bd. I: Manuskripte, Inkunabeln und Druckwerke zu den alphabetisch gereihten Fächergruppen Altertumskunde bis Geschichte. Wien: Finsterbeck.

Becker, Moritz Alois Ritter von (1875): Die Sammlungen der vereinten Familien- und Privatbibliothek Sr. M. des Kaisers. Bd. II/1: Abteilungen Gesellschaft bis Schöne Wissenschaften und Sprachwissenschaften. Wien: Finsterbeck.

Becker, Moritz Alois Ritter von (1879): Die Sammlungen der vereinten Familien- und Privatbibliothek Sr. M. des Kaisers. Bd. II/2: Theologie bis Vereins- und Ordenswesen sowie Nachträge bis Juni 1877, Autoren- und Anonymen-Register. Wien: Verlag Holzhausen.
Becker, Moritz Alois Ritter von (1882): Die Sammlungen der vereinten Familien- und Privatbibliothek Sr. M. des Kaisers. Bd. III/1: Landkarten und topographische Ansichten. Wien: Verlag Holzhausen. Beetz, Wilhelm (1935): Die Porträtsammlung der Nationalbibliothek in ihrer Entwicklung. Zur Erinnerung an die vor 150 Jahren erfolgte Gründung der ehemaligen k. u. k. Familien-Fideikommissbibliothek durch Kaiser Franz I. von Österreich. Graz: Verl. D.-Dt. Vereins-Dr.

Geyer, Rudolf (1901): Vorschrift für die Verfassung des alphabetischen Nominal-Zettelkataloges der Druckwerke der k. k. Hofbibliothek. Wien: Selbstverlag der k. k. Hofbibliothek.

Gilhofer, Ranschburg (1923): Catalogue 157. Rare and valuable books, incunabula, woodcut books, important library-works. Books on the fine arts comprising duplicates of the imperial (national) library Vienna, the library of the counts Inzaghi. Wien: Gilhofer \& Ranschburg.

Huber-Frischeis, Thomas; Knieling, Nina; Valenta, Rainer (2015): Die Privatbibliothek Kaiser Franz` I. von Österreich 1784-1835. Bibliotheks- und Kulturgeschichte einer fürstlichen Sammlung zwischen Aufklärung und Vormärz. Wien: Böhlau (Veröffentlichungen der Kommission für die Neuere Geschichte Österreichs; 111,1).

Mittendorfer, Konstanze (Red.) (1994): Druckschriftenbestand der Österreichischen Nationalbibliothek. 3. Kataloge. Handbuch der historischen Buchbestände in Österreich. Band 1,1, hg. v. Helmut W. Lang, 122-27. Wien, Hildesheim, Zürich, New York: Österreichische Nationalbibliothek.

Payer von Thurn, Rudolf (1927): Ein kaiserlicher Bibliophile. Von Büchern und Menschen. Festschrift Fedor von Zobeltitz zum 5. Oktober 1927 überreicht von der Gesellschaft der Bibliophilen, hg. v. Conrad Höfer, 64-97. Weimar: Ges. der Bibliophilen.

Stummvoll, Josef (Hrsg.) (1968): Zur Geschichte der Österreichischen Nationalbibliothek. Die Hofbibliothek (1368-1922). Wien: Prachner (Museion NF; 2, 3).

Stummvoll, Josef (Hrsg.) (1973): Zur Geschichte der Österreichischen Nationalbibliothek. Die Nationalbibliothek (1923-1967). Wien: Prachner (Museion NF; 2, 3).

Wieser, Walter (1994): Porträtsammlung und Bildarchiv der Österreichischen Nationalbibliothek. Handbuch der historischen Buchbestände in Österreich. Bd. 1,1, hg. v. Helmut W. Lang, 150-55. Wien: Österreichische Nationalbibliothek.

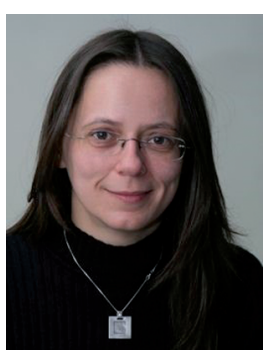

Mag. Michaela Ortner

Österreichische Nationalbibliothek Bildarchiv und Grafiksammlung Leiterin der Abteilung Fideikommissbibliothek/Bestandspflege Josefsplatz 1 A-1015 Wien Österreich michaela.ortner@onb.ac.at 